\title{
低温反应溅射 $\mathrm{Al}+\alpha-\mathrm{Al}_{2} \mathrm{O}_{3}$ 复合靶沉积 $\alpha-\mathrm{Al}_{2} \mathrm{O}_{3}$ 薄膜
}

\author{
程奕天 ${ }^{1,2}$, 邱万奇 ${ }^{1}$, 周克崧 ${ }^{1,2}$, 刘仲武 ${ }^{1}$, 焦东玲 ${ }^{1}$, 钟喜春 ${ }^{1}$, 张 辉 ${ }^{1}$
}

( 1. 华南理工大学 材料科学与工程学院, 广州 510640; 2. 广东省新材料研究所 现代材料表面工程技术国家工 程实验室，广东省现代表面工程技术重点实验室，广州510651）

摘 要: 低温沉积 $\alpha-\mathrm{Al}_{2} \mathrm{O}_{3}$ 薄膜是拓展其实际工程应用的关键。本研究以 $\mathrm{Al} 、 \alpha-\mathrm{Al}_{2} \mathrm{O}_{3}$ 和 $\mathrm{Al}+15 \mathrm{wt} \% \alpha-\mathrm{Al}_{2} \mathrm{O}_{3}$ 为靶材, 用射频磁控溅射在 $\operatorname{Si}(100)$ 基体上沉积氧化铝薄膜。用掠入射 $X$ 射线衍射(GIXRD)、透射电子显微镜(TEM)、能谱 仪(EDS)对所沉积薄膜的相结构和元素含量进行研究, 用纳米压痕技术测量薄膜硬度。结果表明, 在 $550{ }^{\circ} \mathrm{C}$ 的基体 温度下, 反应射频磁控溅射 $\mathrm{Al}+\alpha-\mathrm{Al}_{2} \mathrm{O}_{3}$ 靶可获得单相 $\alpha-\mathrm{Al}_{2} \mathrm{O}_{3}$ 薄膜。靶中的 $\alpha-\mathrm{Al}_{2} \mathrm{O}_{3}$ 溅射至基片表面能优先形成 $\alpha-\mathrm{Al}_{2} \mathrm{O}_{3}$ 晶核, 在 $550{ }^{\circ} \mathrm{C}$ 及以上的基体温度下可抑制 $\gamma$ 相形核, 促进 $\alpha-\mathrm{Al}_{2} \mathrm{O}_{3}$ 晶核同质外延生长, 并最终形成单相 $\alpha-\mathrm{Al}_{2} \mathrm{O}_{3}$ 薄膜。

关 键 词: $\alpha-\mathrm{Al}_{2} \mathrm{O}_{3}$; 反应溅射; 复合靶; 低温沉积; 纳米压痕

中图分类号: TQ174 文献标识码: A

\section{Low-temperature Deposition of $\alpha-\mathrm{Al}_{2} \mathrm{O}_{3}$ Films by Reactive Sputtering $\mathrm{Al}+\alpha-\mathrm{Al}_{2} \mathrm{O}_{3}$ Target}

\author{
CHENG Yi-Tian ${ }^{1,2}$, QIU Wan-Qi ${ }^{1}$, ZHOU Ke-Song ${ }^{1,2}$, LIU Zhong-Wu ${ }^{1}$, JIAO Dong-Ling ${ }^{1}$, \\ ZHONG Xi-Chun ${ }^{1}$, ZHANG Hui ${ }^{1}$
}

(1. School of Materials Science and Engineering, South China University of Technology, Guangzhou 510640, China; 2. The Key Lab of Guangdong for Modern Surface Engineering Technology, National Engineering Laboratory for Modern Materials Surface Engineering Technology, Guangdong Institute of New Materials, Guangzhou 510651, China)

\begin{abstract}
Low-temperature deposition of $\alpha-\mathrm{Al}_{2} \mathrm{O}_{3}$ film is the key to expand its industrial applications. $\mathrm{Al}, \alpha-\mathrm{Al}_{2} \mathrm{O}_{3}$ and $\mathrm{Al}+15 \mathrm{wt} \% \alpha-\mathrm{Al}_{2} \mathrm{O}_{3}$ targets were used to deposit alumina films on $\mathrm{Si}(100)$. The as-deposited films by radio frequency magnetron sputtering (RFMS) were analyzed by grazing incidence X-ray diffraction (GIXRD), transmission electron microscopy (TEM), energy dispersive X-ray spectroscopy (EDS), and the nano-hardness was measured by depth-sensing indentation method. The results show that the single phase $\alpha-\mathrm{Al}_{2} \mathrm{O}_{3}$ films were successfully deposited by reactive sputtering the $\mathrm{Al}+\alpha-\mathrm{Al}_{2} \mathrm{O}_{3}$ composite target at $550{ }^{\circ} \mathrm{C}$. When deposited at the substrate temperature of $550{ }^{\circ} \mathrm{C}$, the $\alpha-\mathrm{Al}_{2} \mathrm{O}_{3}$ sputtered from the target preferentially form $\alpha-\mathrm{Al}_{2} \mathrm{O}_{3}$ nucleus which could suppress the formation of $\gamma$ phase, and promote the homoepitaxial growth of the $\alpha-\mathrm{Al}_{2} \mathrm{O}_{3}$ to obtain the single phase $\alpha-\mathrm{Al}_{2} \mathrm{O}_{3}$ films.
\end{abstract}

Key words: $\alpha-\mathrm{Al}_{2} \mathrm{O}_{3}$; reactive sputtering; composite target; low-temperature deposition; nano-indentation

$\alpha-\mathrm{Al}_{2} \mathrm{O}_{3}$ 具有高温硬度高、化学稳定性好等优良 的综合性能, 是理想的刀具耐磨材料 ${ }^{[1-3]}$ 。氧化铝有
多种同质异构晶体，包括 $\alpha, \gamma, \kappa$ 及非晶等结构 ${ }^{[4-8]}$, 除 $\alpha-\mathrm{Al}_{2} \mathrm{O}_{3}$ 外, 其余均为亚稳相, 硬度也较低, 在

收稿日期：2018-10-10；收到修改稿日期：2019-02-22

基金项目: 国家自然科学基金(51271079); 广东省科技计划项目(2017B030314122); 广州市科技计划项目(201607010091) National Natural Science Foundation of China (51271079); Science and Technology Planning Project of Guangdong Province (2017B030314122); Science and Technology Program of Guangzhou (201607010091)

作者简介: 程奕天(1990-), 男, 博士研究生. E-mail: ytcheng220@gmail.com

通讯作者：邱万奇, 教授. E-mail: mewqqiu@scut.edu.cn 
$1000{ }^{\circ} \mathrm{C}$ 以上时均转变为热力学稳定的 $\alpha-\mathrm{Al}_{2} \mathrm{O}_{3}$, 并 伴随着相变体积变化而导致涂层开裂或崩落 ${ }^{[4]}$ 。因 而只有 $\alpha-\mathrm{Al}_{2} \mathrm{O}_{3}$ 才能用作切削刀具、防扩散层及高 温抗氧化涂层。目前用高温化学气相沉积法可成功 制备出单相的 $\alpha-\mathrm{Al}_{2} \mathrm{O}_{3}$ 涂层 ${ }^{[9]}$, 但高于 $1000{ }^{\circ} \mathrm{C}$ 的沉 积温度极大地限制了基体的选择范围。直接降低沉 积温度通常会导致 $\gamma$ 或其他亚稳相混入氧化铝薄膜 中，显著恶化薄膜的高温稳定性。如何实现 $\alpha-\mathrm{Al}_{2} \mathrm{O}_{3}$ 薄膜的低温沉积一直是众多学者的研究目标。近二 十年来, 众多学者期望使用物理气相沉积(PVD)来 实现 $\alpha-\mathrm{Al}_{2} \mathrm{O}_{3}$ 薄膜的低温沉积。Zywitzki 等 ${ }^{[10-11]}$ 用脉 冲反应磁控溅射在 $760{ }^{\circ} \mathrm{C}$ 时沉积出单相的 $\alpha-\mathrm{Al}_{2} \mathrm{O}_{3}$ 薄膜。Brill 等 ${ }^{[12]}$ 用磁过滤电弧离子镀技术在 $650{ }^{\circ} \mathrm{C}$ 时获得了主相为 $\alpha-\mathrm{Al}_{2} \mathrm{O}_{3}$ 的薄膜。Selinder 等 ${ }^{[13-14]}$ 用高功率脉冲磁控溅射(HiPIMS)系统同样在 $650{ }^{\circ} \mathrm{C}$ 时获得了 $\alpha-\mathrm{Al}_{2} \mathrm{O}_{3}$ 薄膜。

McHale 等 ${ }^{[15]}$ 的研究结果表明, $\gamma-\mathrm{Al}_{2} \mathrm{O}_{3}$ 具有比 $\alpha-\mathrm{Al}_{2} \mathrm{O}_{3}$ 更低的表面能, $\alpha-\mathrm{Al}_{2} \mathrm{O}_{3}$ 晶粒只有在大于 $12 \mathrm{~nm}$ 时在热力学上才比 $\gamma$ 相更稳定, 这需要在较高的温 度才能实现。低温沉积时, 基片表面的氧化铝分子 或分子团簇凝结成的晶核尺寸都很小, 在热力学上 更易形成 $\gamma$ 相, 更低沉积温度时, 则以非晶氧化铝 为主 ${ }^{[16]}$ 。若在基片表面均匀分布有 $\alpha$ 相的籽晶, 氧 化铝分子或分子团簇通过短距离迁移到达籽晶实现 外延生长, 将能有效抑制 $\gamma-\mathrm{Al}_{2} \mathrm{O}_{3}$ 的形核并实现 $\alpha-\mathrm{Al}_{2} \mathrm{O}_{3}$ 的低温生长。 $\alpha-\mathrm{Cr}_{2} \mathrm{O}_{3}$ 与 $\alpha-\mathrm{Al}_{2} \mathrm{O}_{3}$ 结构相同, 晶格常数接近, 且易于在低温下形成, 是用作 $\alpha$ 相 籽晶的理想选择 ${ }^{[17]}$ 。Andersson 等 ${ }^{[18]}$ 在 $\mathrm{Cr}_{2} \mathrm{O}_{3}$ 过渡 层上于 $500{ }^{\circ} \mathrm{C}$ 时沉积出 $\alpha-\mathrm{Al}_{2} \mathrm{O}_{3}$ 薄膜。Eklund 等 ${ }^{[19]}$ 于 $450{ }^{\circ} \mathrm{C}$ 时在 $\alpha-\mathrm{Cr}_{2} \mathrm{O}_{3}$ 过渡层上观察到 $\alpha-\mathrm{Al}_{2} \mathrm{O}_{3}$ 的 外延生长。 Tao 等 ${ }^{[20]}$ 用双辉光溅射沉积出 $\mathrm{Al}+\alpha-\mathrm{Al}_{2} \mathrm{O}_{3}$ 复合薄膜, 后在 $580{ }^{\circ} \mathrm{C}$ 时真空离子热氧 化, 仅在外层获得十纳米的 $\alpha-\mathrm{Al}_{2} \mathrm{O}_{3}$ 膜。从结构上看, $\alpha-\mathrm{Al}_{2} \mathrm{O}_{3}$ 比 $\alpha-\mathrm{Cr}_{2} \mathrm{O}_{3}$ 能更有效地促进 $\alpha-\mathrm{Al}_{2} \mathrm{O}_{3}$ 的外延 生长, 然而至今未见用 $\alpha-\mathrm{Al}_{2} \mathrm{O}_{3}$ 作籽晶在异质基体 上低温沉积 $\alpha-\mathrm{Al}_{2} \mathrm{O}_{3}$ 薄膜的报道。

如何在异质基体表面种植高密度的 $\alpha-\mathrm{Al}_{2} \mathrm{O}_{3}$ 籽 晶是低温沉积 $\alpha-\mathrm{Al}_{2} \mathrm{O}_{3}$ 薄膜的关键。本文对比射频 溅射 $\alpha-\mathrm{Al}_{2} \mathrm{O}_{3}$ 靶和反应射频溅射 $\mathrm{Al}$ 靶沉积的氧化铝 薄膜时发现, 在 $550{ }^{\circ} \mathrm{C}$ 的基体温度时, 反应溅射 $\mathrm{Al}$ 靶只能得到 $\alpha+\gamma$ 双相氧化铝薄膜, 而溅射 $\alpha-\mathrm{Al}_{2} \mathrm{O}_{3}$ 靶则能得到 $\alpha-\mathrm{Al}_{2} \mathrm{O}_{3}$ 薄膜, 推测从 $\alpha-\mathrm{Al}_{2} \mathrm{O}_{3}$ 靶溅射出 的粒子流中部分为含有刚玉结构 $\mathrm{Al}-\mathrm{O}$ 键的分子团 簇, 促进了 $\alpha-\mathrm{Al}_{2} \mathrm{O}_{3}$ 的低温形核。其宏观效果是从 $\alpha-\mathrm{Al}_{2} \mathrm{O}_{3}$ 靶溅射出的部分粒子能形成 $\alpha-\mathrm{Al}_{2} \mathrm{O}_{3}$ 籽晶。 基于上述推测, 本文用自烧结 $\mathrm{Al}+\mathrm{Al}_{2} \mathrm{O}_{3}(15 \mathrm{wt} \%)$ 作
靶材, 用反应射频磁控溅射法成功在 $550{ }^{\circ} \mathrm{C}$ 沉积出 单相的 $\alpha-\mathrm{Al}_{2} \mathrm{O}_{3}$ 薄膜。

\section{1 实验方法}

用射频磁控溅射(RFMS)制备样品, 其示意结构 如图 1 所示。基体底座可在室温至 $750{ }^{\circ} \mathrm{C}$ 间任意调 节。溅射气体 $(\mathrm{Ar})$ 与反应气体 $\left(\mathrm{O}_{2}\right)$ 分别送入沉积室的 靶和基体附近, 以确保大部分氧化反应发生在基体 表面。所有溅射靶材尺寸均为 $\phi 60 \mathrm{~mm} \times 3 \mathrm{~mm}$, 其中 $\mathrm{Al}$ 靶(99.99\%)与 $\alpha-\mathrm{Al}_{2} \mathrm{O}_{3}$ 陶瓷靶(99.9\%)从企业购入, 而 $\mathrm{Al}+\mathrm{Al}_{2} \mathrm{O}_{3}$ 复合靶 $\left(85 \mathrm{wt} \% \mathrm{Al}, 15 \mathrm{wt} \% \alpha-\mathrm{Al}_{2} \mathrm{O}_{3}\right.$ )则用 高纯 $\mathrm{Al}$ 和 $\alpha-\mathrm{Al}_{2} \mathrm{O}_{3}$ 粉用真空热压烧结法自制而成。 采用 $10 \mathrm{~mm} \times 10 \mathrm{~mm}$ 的 $\mathrm{Si}(100)$ 作基体, 在丙酮中超 声清洗 $15 \mathrm{~min}$ 后烘干并置于基体底座上, 靶材与基 体间距固定在 $80 \mathrm{~mm}$ 。抽本底真空至 $5 \times 10^{-4} \mathrm{~Pa}$ 后, 通入高纯 $\mathrm{Ar}$ 与 $\mathrm{O}_{2}$, 流量分别为 12.5 和 $1.25 \mathrm{sccm}$ (溅 射 $\alpha-\mathrm{Al}_{2} \mathrm{O}_{3}$ 靶时不通 $\mathrm{O}_{2}$ ), 沉积气压为 $1 \mathrm{~Pa}$ 。射频电 源功率密度约为 $7.1 \mathrm{~W} / \mathrm{cm}^{2}$ 。沉积 $3 \mathrm{~h}$ 后, 薄膜厚度 约为 $350 \mathrm{~nm}$ 。

用掠入射 X 射线衍射(GIXRD)分析薄膜的相结 构, 掠入射角 $\omega=1^{\circ}$; 用透射电子显微镜(TEM)对微 观相结构进行研究; 用能谱(EDS)对薄膜的元素组 成进行研究; 用纳米压痕仪测量薄膜的显微硬度, 最大压入载荷为 $0.5 \mathrm{mN}$, 保载时间为 $2 \mathrm{~s}$, 每个样品 测试 5 个压痕后取平均值。

\section{2 结果与讨论}

表 1 为 $550{ }^{\circ} \mathrm{C}$ 时浌射 $\mathrm{Al} 、 \alpha-\mathrm{Al}_{2} \mathrm{O}_{3}$ 和 $\mathrm{Al}+\alpha-\mathrm{Al}_{2} \mathrm{O}_{3}$ 靶所沉积薄膜的元素组成。可见 $\mathrm{O}$ 和 $\mathrm{Al}$ 的原子比 约为 1.5, 表明在本实验条件下溅射 $\mathrm{Al} 、 \alpha-\mathrm{Al}_{2} \mathrm{O}_{3}$ 和 $\mathrm{Al}+\alpha-\mathrm{Al}_{2} \mathrm{O}_{3}$ 靶均能沉积出符合化学计量比的 $\mathrm{Al}_{2} \mathrm{O}_{3}$ 薄膜。

图 2 为 $550{ }^{\circ} \mathrm{C}$ 时溅射 $\mathrm{Al}$ 与 $\alpha-\mathrm{Al}_{2} \mathrm{O}_{3}$ 靶沉积薄膜 的 GIXRD 谱。可见反应溅射 $\mathrm{Al}$ 靶得到的是 $\alpha+\gamma$ 双

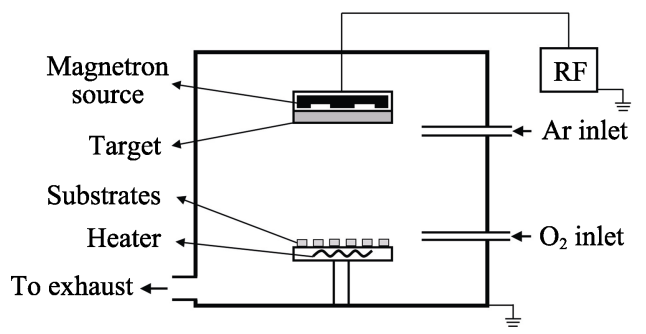

图 1 射频磁控溅射系统的结构原理图

Fig. 1 Schematic diagram of the experimental setup of Radio Frequency (RF) magnetron sputtering system 
表 $1550{ }^{\circ} \mathrm{C}$ 时溅射不同靶材制备的薄膜的元素组成

Table 1 The elementary compositions of the films deposited at $550{ }^{\circ} \mathrm{C}$ from various targets

\begin{tabular}{cccc}
\hline \multirow{2}{*}{ Target } & \multicolumn{3}{c}{ Compositions of the films/at\% } \\
\cline { 2 - 4 } & $\mathrm{Al}$ & $\mathrm{O}$ & $\mathrm{O} / \mathrm{Al}$ \\
\hline $\mathrm{Al}$ & 39.14 & 60.86 & 1.56 \\
$\alpha-\mathrm{Al}_{2} \mathrm{O}_{3}$ & 40.57 & 59.43 & 1.47 \\
$\mathrm{Al}+\alpha-\mathrm{Al}_{2} \mathrm{O}_{3}$ & 38.26 & 61.74 & 1.61 \\
\hline
\end{tabular}

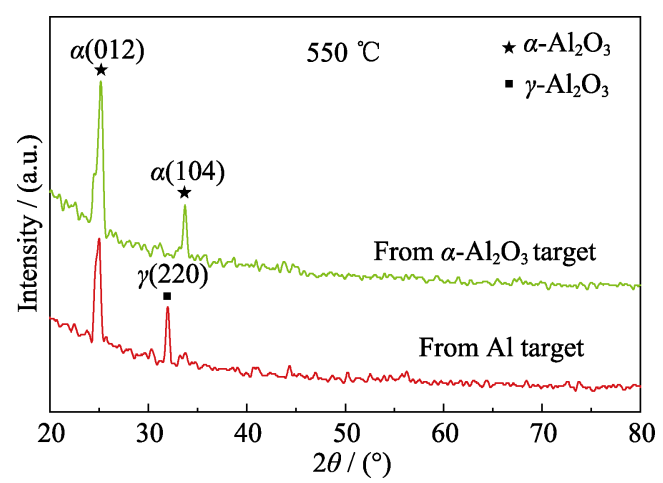

图 $2550{ }^{\circ} \mathrm{C}$ 时溅射 $\mathrm{Al}$ 和 $\alpha-\mathrm{Al}_{2} \mathrm{O}_{3}$ 靶沉积薄膜的 GIXRD 谱图 Fig. 2 GIXRD patterns of the films deposited at $550{ }^{\circ} \mathrm{C}$ from $\mathrm{Al}$ target and $\alpha-\mathrm{Al}_{2} \mathrm{O}_{3}$ target

相氧化铝薄膜, 而溅射 $\alpha-\mathrm{Al}_{2} \mathrm{O}_{3}$ 靶得到的则是 $\alpha-\mathrm{Al}_{2} \mathrm{O}_{3}$ 薄膜。从 $\mathrm{Al}$ 靶溅射出来的 $\mathrm{Al}$ 原子和原子团 簇在基体表面附近被立刻氧化, 形成氧化铝分子和 分子团簇并放出生成热(这些热量有利于氧化铝分 子和分子团簇在基体表面的迁移)。在 $550{ }^{\circ} \mathrm{C}$ 时, 氧 化铝分子和分子团簇在 $\mathrm{Si}(100)$ 基体表面随机迁移 能力较强, 相互碰撞形成岛状晶核。当这些随机碰 撞在一起的氧化铝分子和分子团簇排列结构趋近于 面心立方时, 结晶形成 $\gamma-\mathrm{Al}_{2} \mathrm{O}_{3}$ 晶核; 而当其排列结 构趋近于六方结构时结晶形成 $\alpha-\mathrm{Al}_{2} \mathrm{O}_{3}$ 晶核。形成 $\alpha$ 和 $\gamma$ 晶核后, 晶体释放结晶潜热, 体积增大, 表面迁 移能力降低, 后续的晶核长大更多的是靠基体表面 氧化铝分子和分子团簇迁移到晶核上进行外延生 长。部分氧化铝分子团簇可能难以迁移到邻近晶核 而形成新晶核或非晶混入薄膜中。 $550{ }^{\circ} \mathrm{C}$ 的基体温 度不足以使 $\gamma-\mathrm{Al}_{2} \mathrm{O}_{3}$ 在之后的沉积过程中越过能垒 转变成 $\alpha-\mathrm{Al}_{2} \mathrm{O}_{3}$, 最终获得 $\alpha+\gamma$ 双相和部分非晶的氧 化铝薄膜, 这与 $550{ }^{\circ} \mathrm{C}$ 时热分解勃姆石 $(\mathrm{AlOOH})$ 只 能得到 $\gamma-\mathrm{Al}_{2} \mathrm{O}_{3}$ 有很大不同 ${ }^{[21]}$ 。在同样温度下溅射 $\alpha-\mathrm{Al}_{2} \mathrm{O}_{3}$ 靶所沉积薄膜的 GIXRD 图谱中只有 $\alpha$ 相衍 射峰(图 2), 推测从 $\alpha-\mathrm{Al}_{2} \mathrm{O}_{3}$ 靶溅射出的粒子流中部 分为含有能形成刚玉结构 Al-O 键的分子团簇, 这 些团簇易于凝结成 $\alpha-\mathrm{Al}_{2} \mathrm{O}_{3}$ 晶核, 邻近的氧化铝分
子则迁移到 $\alpha$ 相晶核上形成同质外延生长, 抑制了 $\gamma$ 相的形核并促进了 $\alpha-\mathrm{Al}_{2} \mathrm{O}_{3}$ 的低温生长, 有些难以 迁移到邻近 $\alpha$ 晶核的氧化铝分子将以非晶的形态混 入 $\alpha-\mathrm{Al}_{2} \mathrm{O}_{3}$ 薄膜中。图 2 中的两条 GIXRD 曲线在 $2 \theta$ 角小于 $35^{\circ}$ 时背底强度有明显的升高, 这可能是薄 膜中的非晶相引起的。对图 2 中两条曲线用谢乐公 式计算, 溅射 $\mathrm{Al}$ 靶所沉积薄膜的平均晶粒尺寸为 $\sim 12.9 \mathrm{~nm}$, 而溅射 $\alpha-\mathrm{Al}_{2} \mathrm{O}_{3}$ 靶所沉积的薄膜则为 $\sim 21.4 \mathrm{~nm}$ 。

依据浌射 $\alpha-\mathrm{Al}_{2} \mathrm{O}_{3}$ 靶在基体表面能优先形成 $\alpha$ 相晶核的推测, 本研究用反应射频溅射 $\mathrm{Al}+\alpha-\mathrm{Al}_{2} \mathrm{O}_{3}$ 靶 $\left(85 \mathrm{wt} \% \mathrm{Al}, 15 \mathrm{wt} \% \alpha-\mathrm{Al}_{2} \mathrm{O}_{3}\right)$ 来验证, 并期望实现 在 $550{ }^{\circ} \mathrm{C}$ 低温沉积出单相 $\alpha-\mathrm{Al}_{2} \mathrm{O}_{3}$ 薄膜。图 3 为反 应射频磁控溅射 $\mathrm{Al}+\alpha-\mathrm{Al}_{2} \mathrm{O}_{3}$ 靶所沉积薄膜的 GIXRD 谱, 图中只出现了尖锐的 $\alpha-\mathrm{Al}_{2} \mathrm{O}_{3}$ 衍射峰, 表明所沉积薄膜主要由 $\alpha-\mathrm{Al}_{2} \mathrm{O}_{3}$ 构成。从 $\mathrm{Al}+\alpha-\mathrm{Al}_{2} \mathrm{O}_{3}$ 靶溅射出的粒子流主要有两种: 一种是从复合靶中 的 $\alpha-\mathrm{Al}_{2} \mathrm{O}_{3}$ 溅射出的氧化铝分子和具有 $\alpha$ 型 $\mathrm{Al}-\mathrm{O}$ 键 的氧化铝分子团簇, 这些团簇能在基体表面优先形 成 $\alpha-\mathrm{Al}_{2} \mathrm{O}_{3}$ 晶核, 起到籽晶的作用。另一种是从复合 靶中的 $\mathrm{Al}$ 溅射出的 $\mathrm{Al}$ 原子和原子团簇, 在基体表 面立刻氧化形成氧化铝分子和分子团簇, 并释放出 生成热。在 $550{ }^{\circ} \mathrm{C}$ 基体温度和额外生成热的促进下, 这些粒子能迁移到邻近的 $\alpha$ 相晶核进行外延生长, 最终形成单相 $\alpha-\mathrm{Al}_{2} \mathrm{O}_{3}$ 薄膜。图 3 中插入了溅射 $\alpha-\mathrm{Al}_{2} \mathrm{O}_{3}$ 靶所沉积薄膜的 GIXRD 谱, 可见用 $\mathrm{Al}+\alpha-\mathrm{Al}_{2} \mathrm{O}_{3}$ 复合靶沉积的薄膜中 $\alpha$ 相的衍射峰比用 $\alpha-\mathrm{Al}_{2} \mathrm{O}_{3}$ 靶沉积的薄膜更尖锐, 表明薄膜中的 $\alpha-\mathrm{Al}_{2} \mathrm{O}_{3}$ 晶粒更大, 非晶相更少, 用谢乐公式计算出 的平均晶粒尺寸为 $26.3 \mathrm{~nm}$ 。这可能是由于在同样 存在 $\alpha$ 相籽晶的情况下, 反应溅射 $\mathrm{Al}+\alpha-\mathrm{Al}_{2} \mathrm{O}_{3}$ 复合

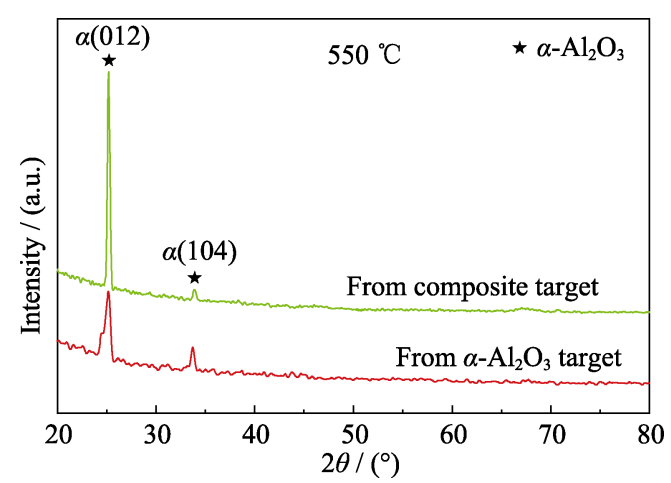

图 $3550{ }^{\circ} \mathrm{C}$ 时溅射 $\alpha-\mathrm{Al}_{2} \mathrm{O}_{3}$ 靶和 $\mathrm{Al}+\alpha-\mathrm{Al}_{2} \mathrm{O}_{3}$ 复合靶沉积薄 膜的 GIXRD 图谱

Fig. 3 GIXRD patterns of the film deposited at $550{ }^{\circ} \mathrm{C}$ from $\alpha-\mathrm{Al}_{2} \mathrm{O}_{3}$ target and $\mathrm{Al}+\alpha-\mathrm{Al}_{2} \mathrm{O}_{3}$ composite target 
靶的过程中 $\mathrm{Al}$ 在基体表面的氧化生成热增强了氧 化铝粒子的表面迁移，进而促进了 $\alpha-\mathrm{Al}_{2} \mathrm{O}_{3}$ 的结晶 生长, 并减少了非晶氧化铝的含量。用 $\mathrm{Al}+\alpha-\mathrm{Al}_{2} \mathrm{O}_{3}$ 复合靶替代 $\alpha-\mathrm{Al}_{2} \mathrm{O}_{3}$ 靶沉积 $\alpha-\mathrm{Al}_{2} \mathrm{O}_{3}$ 薄膜, 具有实际 工程应用价值, 因大面积溅射沉积需要大面积靶材, 而脆性的 $\alpha-\mathrm{Al}_{2} \mathrm{O}_{3}$ 大面积靶材很容易因热振冲击而 碎裂, $\mathrm{Al}+\alpha-\mathrm{Al}_{2} \mathrm{O}_{3}$ 复合靶的韧性要好得多, 能够确 保制成大面积靶材在沉积时的稳定性。

基体温度同样显著影响着薄膜的相结构组成。 图 4 为 $500{ }^{\circ} \mathrm{C}$ 时反应溅射 $\mathrm{Al}+\alpha-\mathrm{Al}_{2} \mathrm{O}_{3}$ 复合靶所沉积 薄膜的 GIXRD 谱。图中除出现 $\alpha$ 相的(012)和(104) 衍射峰外, 还有 $\gamma-\mathrm{Al}_{2} \mathrm{O}_{3}$ 的(220)衍射峰。在低角度较 高的背底也表明薄膜中还含有较多的非晶相。虽然 $\alpha-\mathrm{Al}_{2} \mathrm{O}_{3}$ 晶核仍能在基体表面形成，但因基体温度 低, 较远距离的氧化铝难以迁移到邻近的 $\alpha$ 相晶核 进行同质外延生长, 只能随机堆叠成核, 部分形成 $\gamma$ 相和非晶相。上述结果表明, 在射频磁控溅射系统 中溅射 $\mathrm{Al}+\alpha-\mathrm{Al}_{2} \mathrm{O}_{3}$ 复合靶制备氧化铝薄膜时, 需 $550{ }^{\circ} \mathrm{C}$ 或以上的温度才能形成单相的 $\alpha-\mathrm{Al}_{2} \mathrm{O}_{3}$ 薄膜。

图 5(a)为 $550{ }^{\circ} \mathrm{C}$ 时溅射 $\alpha-\mathrm{Al}_{2} \mathrm{O}_{3}$ 靶沉积氧化铝 薄膜靠近基体附近的 TEM 照片, 两插图分别为方框 区域的放大图及其快速傅里叶变换(FFT)花样。由插 图及其对应的 FFT 可推断出方框中晶粒为 $\alpha-\mathrm{Al}_{2} \mathrm{O}_{3}$, 其周围的是非晶相。图 5(b)为反应浌射 $\mathrm{Al}+\alpha-\mathrm{Al}_{2} \mathrm{O}_{3}$ 复合靶所沉积薄膜靠近基体表面附近的 TEM 照片。 图中仅可标定出 $\alpha-\mathrm{Al}_{2} \mathrm{O}_{3}$, 未发现非晶相或其他亚稳 相的存在, 说明该薄膜主要由 $\alpha-\mathrm{Al}_{2} \mathrm{O}_{3}$ 构成, 与图 3 中的 GIXRD 结果一致。

图 6(a)为分别溅射 $\mathrm{Al} 、 \alpha-\mathrm{Al}_{2} \mathrm{O}_{3}$ 及 $\mathrm{Al}+\alpha-\mathrm{Al}_{2} \mathrm{O}_{3}$ 靶所沉积薄膜的载荷-位移(P-h)曲线。图 6(b)为依据 图 6(a)中数据用 Oliver-Pharr 公式 ${ }^{[22]}$ 计算得到的平 均硬度。溅射 $\mathrm{Al} 、 \alpha-\mathrm{Al}_{2} \mathrm{O}_{3}$ 及 $\mathrm{Al}+\alpha-\mathrm{Al}_{2} \mathrm{O}_{3}$ 靶所沉积

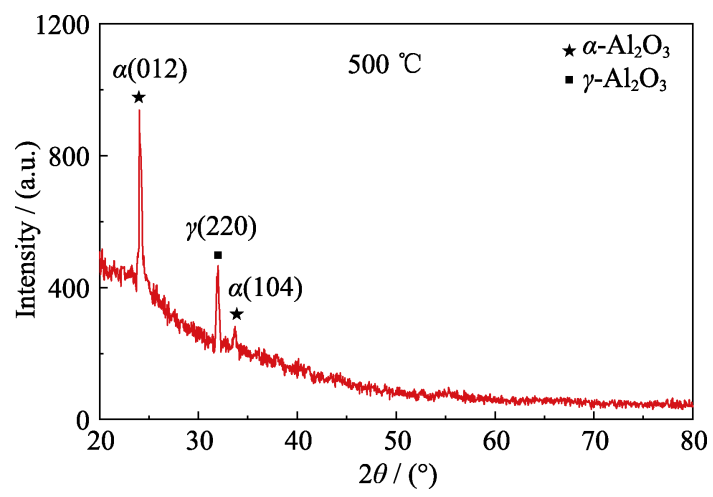

图 $4500{ }^{\circ} \mathrm{C}$ 溅射 $\mathrm{Al}+\alpha-\mathrm{Al}_{2} \mathrm{O}_{3}$ 复合靶沉积薄膜的 GIXRD 图谱 Fig. 4 GIXRD pattern of the film deposited from $\mathrm{Al}+\alpha-\mathrm{Al}_{2} \mathrm{O}_{3}$ composite target at $500{ }^{\circ} \mathrm{C}$
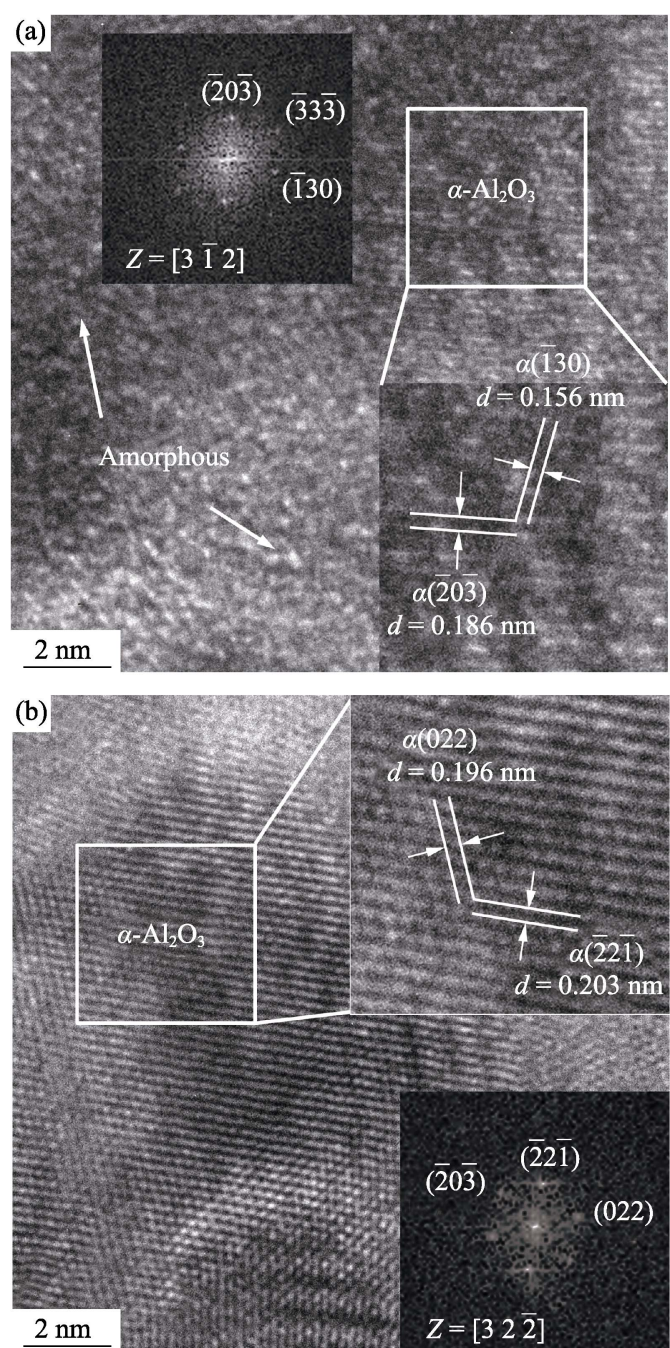

图 $5550{ }^{\circ} \mathrm{C}$ 溅射(a) $\alpha-\mathrm{Al}_{2} \mathrm{O}_{3}$ 靶和(b) $\mathrm{Al}+\alpha-\mathrm{Al}_{2} \mathrm{O}_{3}$ 复合靶沉积 薄膜的 TEM 照片

Fig. 5 TEM micrographs of the films deposited at $500{ }^{\circ} \mathrm{C}$ from (a) $\alpha-\mathrm{Al}_{2} \mathrm{O}_{3}$ and (b) $\mathrm{Al}+\alpha-\mathrm{Al}_{2} \mathrm{O}_{3}$ composite targets

薄膜的硬度 $(H)$ 分别为 16.3, 20.2 和 $23.8 \mathrm{GPa}$ 。薄 膜中相的种类及其相对含量决定了薄膜的硬度。溅 射 $\mathrm{Al}$ 靶沉积的薄膜为 $\alpha+\gamma$ 及非晶相氧化铝, 溅射 $\alpha-\mathrm{Al}_{2} \mathrm{O}_{3}$ 靶沉积的薄膜为 $\alpha$ 以及非晶相氧化铝, 而溅 射 $\mathrm{Al}+\alpha-\mathrm{Al}_{2} \mathrm{O}_{3}$ 复合靶沉积的薄膜以 $\alpha-\mathrm{Al}_{2} \mathrm{O}_{3}$ 相为主。 非晶、 $\gamma$-和 $\alpha-\mathrm{Al}_{2} \mathrm{O}_{3}$ 的硬度分别为 10,19 和 $22 \mathrm{GPa}^{[11]}$ 。 浅射 $\mathrm{Al}$ 靶所沉积薄膜的硬度只有 16.3 GPa，这是 薄膜中非晶和 $\gamma-\mathrm{Al}_{2} \mathrm{O}_{3}$ 含量较多所致; 溅射 $\alpha-\mathrm{Al}_{2} \mathrm{O}_{3}$ 靶所沉积薄膜的硬度为 $20.2 \mathrm{GPa}$, 这是由于薄膜中 少量非晶氧化铝降低了硬度; 溅射 $\mathrm{Al}+\alpha-\mathrm{Al}_{2} \mathrm{O}_{3}$ 复合 靶所沉积薄膜的硬度达 23.8 GPa, 表明薄膜主要由 高硬度的 $\alpha-\mathrm{Al}_{2} \mathrm{O}_{3}$ 组成, 所测量的硬度比文献[11]所 述略高, 这可能是载荷较低而造成的测量误差所致, 也可能有纳米强化的因素。硬度分析结果也从侧面 证明溅射 $\mathrm{Al}+\alpha-\mathrm{Al}_{2} \mathrm{O}_{3}$ 复合靶沉积的薄膜基本由单相 的 $\alpha-\mathrm{Al}_{2} \mathrm{O}_{3}$ 组成。 

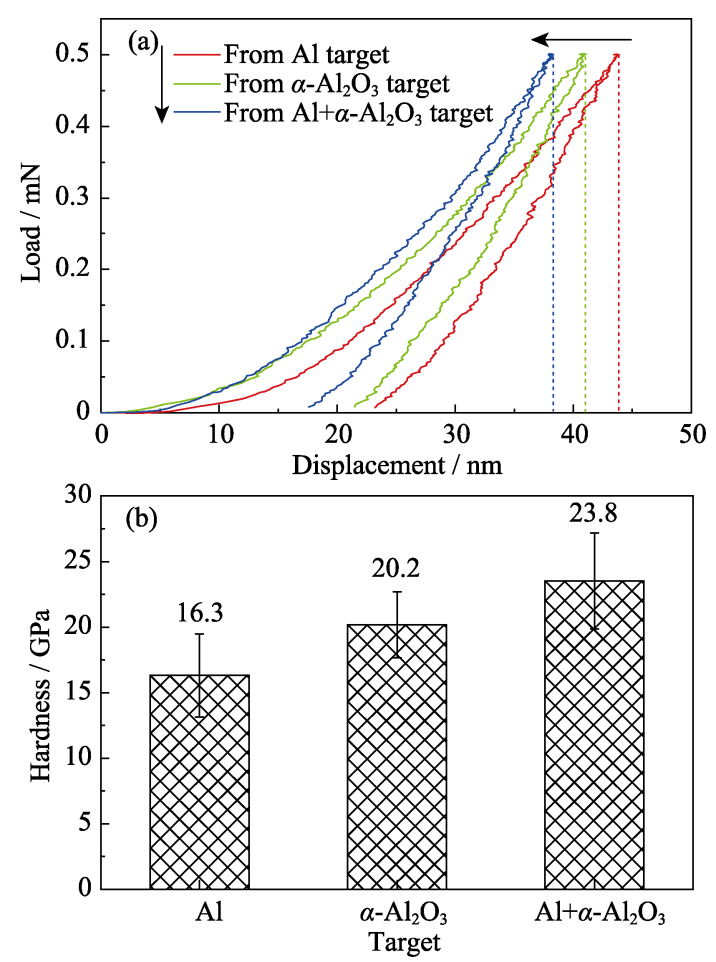

图 $6550{ }^{\circ} \mathrm{C}$ 的基体温度下溅射三种不同靶材沉积薄膜的(a) P-h 曲线和(b)硬度

Fig. 6 (a) P-h curves and (b) Hardness of the alumina films deposited from different targets at $550{ }^{\circ} \mathrm{C}$

\section{3 结论}

1) 射频磁控溅射 $\alpha-\mathrm{Al}_{2} \mathrm{O}_{3}$ 靶, 在 $550{ }^{\circ} \mathrm{C}$ 时能获 得 $\alpha-\mathrm{Al}_{2} \mathrm{O}_{3}$ 为主和少量非晶相的氧化铝薄膜, 薄膜的 硬度为 $20.2 \mathrm{GPa}$;

2) 反应射频磁控溅射 $\mathrm{Al}+\alpha-\mathrm{Al}_{2} \mathrm{O}_{3}(15 \mathrm{wt} \%)$ 复合 靶, 在 $550{ }^{\circ} \mathrm{C}$ 时沉积出纳米单相 $\alpha-\mathrm{Al}_{2} \mathrm{O}_{3}$ 薄膜, 薄膜 硬度为 $23.8 \mathrm{GPa}$;

3) 同时确保基体表面分布有较高密度的 $\alpha$ 相籽 晶和 $550{ }^{\circ} \mathrm{C}$ 或以上的基体温度是反应浌射 $\mathrm{Al}+\alpha-\mathrm{Al}_{2} \mathrm{O}_{3}$ 复合靶沉积单相 $\alpha-\mathrm{Al}_{2} \mathrm{O}_{3}$ 薄膜的基本要求。

\section{参考文献:}

[1] BOUZAKIS K D, MICHAILIDIS N, SKORDARIS G, et al. Cutting with coated tools: coating technologies, characterization methods and performance optimization. CIRP Ann. Manuf. Technol., 2012, 61(2): 703-723.

[2] BOBZIN K, BAGCIVAN N, EWERING M. Crystalline gamma-alumina deposited in an industrial coating unit for demanding turning operations. Adv. Eng. Mater., 2010, 12(1/2): 75-79.

[3] BOBZIN K, HIRT G, BAGCIVAN N, et al. Crystalline gamma- $\mathrm{Al}_{2} \mathrm{O}_{3}$ physical vapor deposition-coating for steel thixoforging tools. J. Nanosci. Nanotechnol., 2011, 11(10): 8782-8785.

[4] EDLMAYR V, MOSER M, WALTER C, et al. Thermal stability of sputtered $\mathrm{Al}_{2} \mathrm{O}_{3}$ coatings. Surf. Coat. Technol., 2010, 204(9/10):
1576-1581.

[5] EKLUND P, SRIDHARAN M, SINGH G, et al. Thermal stability and phase trans- formations of gamma-/amorphous- $\mathrm{Al}_{2} \mathrm{O}_{3}$ thin films. Plasma Process. Polym., 2009, 6: S907-S911.

[6] MUSIL J, BLAZEK J, ZEMAN P, et al. Thermal stability of alumina thin films containing gamma- $\mathrm{Al}_{2} \mathrm{O}_{3}$ phase prepared by reactive magnetron sputtering. Appl. Surf. Sci., 2010, 257(3): 1058-1062.

[7] EDLMAYR V, HARZER T P, HOFFMANN R, et al. Effects of thermal annealing on the microstructure of sputtered $\mathrm{Al}_{2} \mathrm{O}_{3}$ coatings. J. Vac. Sci. Technol. A, 2011, 29(4): 041506-041508.

[8] BOBZIN K, BAGCIVAN N, REINHOLDT A, et al. Thermal stability of gamma- $\mathrm{Al}_{2} \mathrm{O}_{3}$ coatings for challenging cutting operations. Surf. Coat. Technol., 2010, 205(5): 1444-1448.

[9] RUPPI S. Deposition, microstructure and properties of texturecontrolled CVD $\alpha-\mathrm{Al}_{2} \mathrm{O}_{3}$ coatings. Int. J. Refract. Met. Hard Mater. 2005, 23(4/5/6): 306-316.

[10] ZYWITZKI O, HOETZSCH G, FIETZKE F, et al. Effect of the substrate temperature on the structure and properties of $\mathrm{Al}_{2} \mathrm{O}_{3}$ layers reactively deposited by pulsed magnetron sputtering. Surf. Coat. Technol., 1996, 82(1/2): 169-175.

[11] ZYWITZKI O, HOETZSCH G. Influence of coating parameters on the structure and properties of $\mathrm{Al}_{2} \mathrm{O}_{3}$ layers reactively deposited by means of pulsed magnetron sputtering. Surf. Coat. Technol., 1996, 86-87(1/2/3): 640-647.

[12] BRILL R, KOCH F, MAZURELLE J, et al. Crystal structure characterization of filtered arc deposited alumina coatings: temperature and bias voltage. Surf. Coat. Technol., 2003, 174-175: 606-610.

[13] SELINDER T J, CORONEL E, WALLIN E, et al. $\alpha$-Alumina coatings on WC/Co substrates by physical vapor deposition. Int. J. Refract. Met. Hard Mater., 2009, 27(2): 507-512.

[14] WALLIN E, SELINDER T J, ELFWING M, et al. Synthesis of $\alpha-\mathrm{Al}_{2} \mathrm{O}_{3}$ thin film using reactive high-power impulse magnetron sputtering. Europhys. Lett., 2008, 82(3): 36002.

[15] MCHALE J M, AUROUX A, PERROTTA A J, et al. Surface energies and thermo-dynamic phase stability in nanocrystalline alumina. Science, 1997, 277(5327): 788-792.

[16] GAVRILOV N V, KAMENETSKIKH A S, TRETNIKOV P V, et al. Ion assisted deposition of $\alpha-\mathrm{Al}_{2} \mathrm{O}_{3}$ coatings by anodic evaporation in the arc discharge. Surf. Coat. Technol., 2018, 337: 453-460.

[17] JIN P, XU G, TAZAWA M, et al. Low temperature deposition of $\alpha-\mathrm{Al}_{2} \mathrm{O}_{3}$ thin films by sputtering using a $\mathrm{Cr}_{2} \mathrm{O}_{3}$ template. J. Vac. Sci. Technol. A, 2002, 20(6): 2134-2136.

[18] ANDERSSON J M, WALLIN E, HELMERSSON U, et al. Phase control of $\mathrm{Al}_{2} \mathrm{O}_{3}$ thin films grown at low temperature. Thin Solid Films, 2006, 513(1/2): 57-59.

[19] EKLUND P, SRIDHARAN M, SILLASSEN M, et al. $\alpha-\mathrm{Cr}_{2} \mathrm{O}_{3}$ template-texture effect on $\alpha-\mathrm{Al}_{2} \mathrm{O}_{3}$ thin-film growth. Thin Solid Films, 2008, 516(21): 7447-7450.

[20] LIN Y B, WANG C, TAO J. Induction effect of $\alpha-\mathrm{Al}_{2} \mathrm{O}_{3}$ seeds on formation of alumina coatings prepared by glow plasma technique. Surf. Coat. Technol., 2013, 235: 544-551.

[21] ZHANG L, ZHU Y J. Microwave-assisted solvothermal synthesis of $\mathrm{AlOOH}$ hierarchically nanostructured microspheres and their transformation to $\gamma-\mathrm{Al}_{2} \mathrm{O}_{3}$ with similar morphologies. J. Phys. Chem. C, 2008, 112(43): 16764-16768.

[22] OLIVER W C, PHARR G M J. An improved technique for determining hardness and elastic modulus using load and displacement sensing indentation. J. Mater. Res., 1992, 7(6): 1564-1576. 